\title{
Whipple procedure: patient selection and special considerations
}

This article was published in the following Dove Press journal:

Open Access Surgery

27 July 2016

Number of times this article has been viewed

\author{
Clara Tan-Tam' \\ Maja Segedi² \\ Stephen W Chung ${ }^{2}$ \\ 'Department of Surgery, Bassett \\ Healthcare, Columbia University, \\ Cooperstown, New York, NY, USA; \\ ${ }^{2}$ Department of Hepatobiliary \\ and Pancreatic Surgery and Liver \\ Transplant, Vancouver General \\ Hospital, University of British \\ Columbia, Vancouver, BC, Canada
}

Correspondence: Clara Tan-Tam Bassett Healthcare, Columbia University, I Atwell Road, Cooperstown, New York, 13326, USA

Tel +6075474507

Fax +6075476553

Email Clara.tan-tam@bassett.org
Abstract: At the inception of pancreatic surgery by Dr Whipple in 1930s, the mortality and morbidity risk was more than $20 \%$. With further understanding of disease processes and improvements in pancreas resection techniques, the mortality risk has decreased to less than 5\%. Age and chronic illnesses are no longer a contraindication to surgical treatment. Life expectancy and quality of life at a later age have improved, making older patients more likely to receive pancreatic surgery, thereby also putting emphasis on operative patient selection to minimize complications. This review summarizes the benign and malignant illnesses that are treated with pancreas operations, and innovations and improvements in pancreatic surgery and perioperative care, and describes the careful selection process for patients who would benefit from an operation. These indications are not reserved only to Whipple operation, but to pancreatectomies as well.

Keywords: pancreaticoduodenectomy, mortality, morbidity, cancer, trauma, pancreatitis

\section{Introduction}

Whipple pancreaticoduodenectomy (PD) is not performed exclusively for neoplasia but also for benign disease. ${ }^{1}$ In addition to Whipple resection, there are other pancreatic resections such as distal, subtotal, and total pancreatectomy for other types of pancreatic pathology and neoplasia.

The goal of PD is to prevent and treat cancer, and treat disease symptoms. Perioperative care addresses only some of the factors that determine morbidities, decrease hospital stay, and minimize delay to adjuvant chemotherapy. Thus, patient selection is critical. All patients should be evaluated by a hepatobiliary and pancreatic surgeon to determine if they would benefit from a PD. In order to further advance the understanding and treatments of pancreatic diseases, all patients should be encouraged to be part of a registry such as the National Familial Pancreas Tumor Registry.

Medical complications due to surgery include cardiac events, cerebrovascular accidents, respiratory distress, pneumonia, pulmonary embolism, renal dysfunction, and hepatic and metabolic dysfunction. The risk of these complications has decreased substantially. The postoperative medical complication rate is $4 \%-19 \%{ }^{2}$ Overall, the mortality risk due to pancreatic surgery is less than $5 \% .^{3}$

\section{Factors associated with postoperative complications}

A number of factors are associated with perioperative outcomes after PD or Whipple procedure. After adjusting for potential confounders, a study of 4,945 patients who 
underwent PD from 2005 to 2009 from the American College of Surgeons National Surgical Quality improvement database demonstrates that the significant predictors of morbidity included older age, male sex, being overweight and obese, dependent functional status, chronic obstructive pulmonary disease (COPD), steroid use, bleeding disorder, leukocytosis, elevated serum creatinine, and hypoalbuminemia. ${ }^{4}$ Significant predictors of 30-day mortality included COPD, hypertension, neoadjuvant radiation therapy, elevated serum creatinine, and hypoalbuminemia. Japanese studies have demonstrated that risk factors for in-hospital mortality were age, respiratory distress, activities of daily living within 30 days before surgery, angina, weight loss of more than $10 \%$, American Society of Anesthesiologist class greater than 3, Brinkman index of more than 400, body mass index (BMI) of more than $25 \mathrm{~kg} / \mathrm{m}^{2}$, white blood cell count of more than 11,000 cells $/ \mu \mathrm{L}$, platelet count of less than $120,000 / \mu \mathrm{L}$, prothrombin time/international normalized ratio of more than 1.1, activated partial thromboplastin time of more than 40 seconds, and serum creatinine levels of more than $3 \mathrm{mg} / \mathrm{dL}$. ${ }^{4}$ Male sex, emergency surgery, COPD, bleeding disorders, and blood urea nitrogen less than $8 \mathrm{mg} / \mathrm{dL}$ were independent variables in the 30-day mortality group. ${ }^{4}$

Although age is a risk factor, current studies suggest that PD is an acceptable option for elderly patients. ${ }^{5}$ In a review of outcomes of PD completed on 385 patients, 23 patients who were 80 years or older were assessed from 1998 to 2011. When comparing younger patients versus those $>80$ years of age, the study demonstrated that complication rate $(40 \%$ vs $43 \%$ ), mortality rate ( $4 \%$ vs $0 \%$ ), and overall median survival for pancreatic cancer patients were not statistically different between the groups. ${ }^{5}$

Langan et $\mathrm{al}^{6,7}$ assessed 99 elderly patients from 2005 to 2013 and demonstrated that increased age was associated with increased intensive care unit stay, length of stay, and discharge to a skilled facility for rehabilitation. However, octogenarians had equivalent charges and outcome measure when compared with septuagenarians. ${ }^{6}$ They also suggest that it is possible to deliver quality pancreatic surgical care to an aging population without substantial increase in resource utilization. ${ }^{7}$

Rather than chronologic age, it is more important to consider patient's performance status and comorbidities and base the decision to operate on a careful and individual risk-benefit analysis.

\section{BMI}

Overweight patients (BMI $>30 \mathrm{~kg} / \mathrm{m}^{2}$ ) should not be precluded from undergoing Whipple, but they have an increased risk of postoperative morbidity. The mortality rates for patients who are underweight and obese are higher for almost all procedures. ${ }^{8}$

Looking at PDs, some groups have shown that there is increased visceral fat specifically associated with increased postoperative morbidity. ${ }^{9} \mathrm{~A}$ high $\mathrm{BMI}$ is a prognostic marker that portends an abbreviated survival following PD for pancreatic adenocarcinoma, and this is independent of the complexity of the surgery. ${ }^{10}$

Generalized obesity is associated with postoperative wound infections after PD. The degree of visceral fat on preoperative cross-sectional imaging is associated with significantly higher rates of overall complications and pancreatic fistula. ${ }^{11}$ Patients with BMI equal to or greater than $30 \mathrm{~kg} / \mathrm{m}^{2}$ had more wound infections, pancreatic fistula, or abscess. Despite the risks with obesity, being overweight should not be a contraindication for undergoing PD, but meticulous consultation and evaluation of these patients is critical.

In a review of 408 patients who underwent PD for pancreatic adenocarcinoma, Pausch et $\mathrm{al}^{12}$ found that patients with low BMI $\left(<18.5 \mathrm{~kg} / \mathrm{m}^{2}\right)$ and cachexia (unintentional weight loss, malnutrition, and systemic inflammation) had a greater 90 -day mortality $(20 \%$ vs $0 \%)$ and a trend toward greater complication rates and in-hospital mortality $(6.67 \%$ vs $0 \%$ ), despite a greater comorbidity in obese patients with a higher BMI. Patients with BMI between 18.5 and $30 \mathrm{~kg} / \mathrm{m}^{2}$ had a 90 -day mortality of $7.25 \%$ and an in-hospital mortality of $4.83 \%$. Patients with larger amounts of abdominal wall fat had fewer intra-abdominal abscesses, shorter hospital stay, and lower 90-day mortality rate, with a better long-term survival. In pancreatic cancer, underweight patients have a poor outcome after PD than obese patients. ${ }^{11,12}$

Severe nutritional risk predicts decreased long-term survival in geriatric patients undergoing PD for benign disease. Nonoperative management should be considered in geriatric patients with severe nutritional risks when malignancy is not suspected. In general, continued nutritional support is important for recovery.

Being underweight should not be a contraindication for undergoing PD, but pre- and perioperative therapies should be used to optimize the recovery of these patients. In addition, indications, alternatives, and increased risk of morbidity and mortality should be discussed with the patient during the informed consent process, including careful consideration of risks and benefits of surgery at hand.

\section{Anatomical anomalies}

The presence of anatomical anomalies has not been considered a contraindication for surgery. Frequently, PDs are performed on 
patients with aberrant right hepatic arteries and low bifurcating arteries. These are reviewed in numerous textbooks, and many vascular reconstructions are also reviewed by Clancy. ${ }^{13}$

\section{Previous surgeries}

Given the obesity epidemic in the USA, where approximately $7 \%$ of the population is morbidly obese, surgery for weight loss in adults and adolescents is an increasingly popular treatment for this problem. ${ }^{14}$ As this population gets older and an increasing number of pancreatic lesions are found, the hepatopancreaticobiliary (HPB) surgeon will likely encounter more patients with prior bariatric surgery as well as other types of abdominal surgery. Hatzaras et $\mathrm{al}^{15}$ reviewed the challenges and available techniques for reconstruction of patients who undergo PD after bariatric surgery.

The separation of the pancreas to the hepatic arteries includes ligation and division of the gastroduodenal artery. Matthews et al (submitted) describe a PD on a patient for pancreatic adenocarcinoma, who had an open esophagectomy and transhiatal gastric pull-up for esophageal adenocarcinoma. The patient continues to have a good quality of life with no evidence of recurrence to date.

\section{Conclusion}

In summary, as medicine continues to advance, better early detection programs are implemented and there are better treatments for chronic illnesses, the aging population will increase, and age will no longer be a contraindication for surgery for curative intent. Therefore, meticulous perioperative evaluation, rehabilitation and postoperative care of the patient must continue to play a critical role in improving the survivorship of these patients.

\section{Minimally invasive surgery Role of exploratory laparoscopy}

Contraindications to a PD for treatment of cancer include distant metastasis, extrapancreatic disease such as peritoneal implants, vascular invasion, and metastatic lymphadenopathy beyond limits of margin-negative resection. To prevent unnecessary laparotomy, these problems can be identified with imaging and diagnostic laparoscopy. ${ }^{13}$ The value of laparoscopy is controversial as one has to weigh the risks of subjecting the patient to an added intervention and cost. Occult metastasis has been identified in up to $30 \%$ of patients using diagnostic laparoscopy. ${ }^{1,16}$ Because most metastasis have been found in patients with locally invasive disease, ${ }^{17,18}$ some authorities have advocated selective use of diagnostic laparoscopy such as tumors larger than $3 \mathrm{~cm}^{17}$, all lesions of the neck, body and tail of the pancreas ${ }^{18}$, and an elevated $\mathrm{Ca}$ 19-9. ${ }^{17,19}$

Because the incidence of pancreatic cancer and cancers involving the pancreatic head and duodenum is not high, and a diagnostic laparoscopy neither requires too much operative resources nor imposes too much of a risk, some have suggested that a diagnostic laparoscopy should be completed in all patients. ${ }^{13}$

\section{Role of laparoscopic pancreas resection}

Minimally invasive or laparoscopic distal pancreatectomy is a common operation, but minimally invasive PD is not as common. Minimally invasive approaches are a good option for patients with small, low-grade, or benign lesions that are neither near the neck of the pancreas and uncinate process, nor close to the celiac trunk. ${ }^{20}$ High BMI does not appear to be a contraindication. ${ }^{20}$

\section{Surgery for precancerous lesions}

In the past, pancreatic neoplasms were not considered for population screening programs owing to their aggressive biology, rapid progression, and presumed low prevalence. It is understood that pancreatic cancer undergoes a multistep process of increasing grades of dysplasia, and this process takes approximately 10 years before the neoplastic lesion becomes cancer. ${ }^{21,22}$ As imaging techniques improve, neoplastic precursor lesions can be detected at an early stage, possibly decreasing the mortality from pancreatic cancer when screening programs are aimed at high-risk populations. Although they may not be cost-effective at this time, these programs continue to be evaluated.

There are genetic and epigenetic factors, or hereditary and nonhereditary conditions associated with pancreatic cancer. The most significant risk factors for the development of pancreatic cancer are age, with a rapid rise in incidence after the age of 50 years, and smoking. A few, rare genetic disorders contribute up to $10 \%-15 \%$ of the cases..$^{21,22}$ The following diseases have demonstrated an association with an increased risk of pancreatic cancer. These diseases include Peutz-Jeghers polyposis, hereditary pancreatitis, familial atypical multiple mole melanoma, Lynch syndrome or hereditary non-polyposis colonic cancer, hereditary breast-ovarian cancer, familial adematous polyposis, Li-Fraumeni, and familial pancreatic cancer (Table 1). These diseases carry an increased risk in pancreatic cancer and should be part of a screening program. ${ }^{21-23}$

The gene or genes causing familial pancreatic cancer have not been identified, but it appears to be an autosomal dominant transmission with reduced penetrance. The relative 
Table I Hereditary syndromes associated with pancreas cancer, which are an indication for screening

I. Peutz-Jeghers polyposis

(STK II gene, I32-fold risk of pancreas cancer)

2. Hereditary pancreatitis

(PRSSI, SPINKI, CFTR genes; 28-87-fold risk)

3. Familial atypical multiple mole melanoma (FAMMM)

(CDKN2A; 20-47-fold risk)

4. Lynch syndrome/hereditary non-polyposis colonic cancer

(MLH, MSH2 genes; 9-II-fold risk)

5. Hereditary breast-ovarian cancer

(BRCAI, BRCA2 genes; 2.4-6-fold risk)

6. Familial adematous polyposis

(APC gene; 4-5 RR)

7. Li-Fraumeni ${ }^{23}$

(p53 gene; 7.3 RR)

8. Familial pancreatic cancer ${ }^{22}$

(two first-degree relatives)

risk increases from 4.5-fold in case of a single affected firstdegree relative, to 32 -fold in the cases with three or more first-degree relatives. ${ }^{21,22}$

There are currently no screening programs for nonhereditary conditions, which can be associated with an estimated twofold increased risk. This would include new-onset type 2 diabetes, type 1 diabetes, smoking, alcohol abuse, being overweight, exposure to nickel, and previous gastric ulcer. Chronic pancreatitis is associated with a 14-fold increase risk of pancreatic cancer. ${ }^{24}$ Patients with these factors should be considered as higher risk and offered resection on a more urgent basis.

\section{Pancreatic metastases considered for surgery}

It is important to weigh the risk and benefits of performing a pancreatic metastasectomy and improving prognosis of cancer. The benefit and effectiveness of resection for pancreatic metastasis is dependent on the tumor biology of the primary cancer. Recently, an increasingly aggressive attitude toward treatment and an increase in the safety of pancreatic surgery has led to the more common surgical management of pancreatic metastases. A literature review shows that reports of pancreatic resection for isolated metastasis to the pancreas include renal cell carcinoma (RCC), colorectal cancer, melanoma, ovarian, prostate, breast, lungs, and sarcoma. ${ }^{25}$

\section{$\mathrm{RCC}$}

RCC accounts for $0.25 \%-3 \%$ of resected pancreatic tumors. The 5-year survival rate in these cases was as high as $88 \%$, with a 5 -year disease-free survival rate of $57.0 \%{ }^{25}$ Prognostic factors after pancreatic metastasectomy for RCC include tumor grade of the primary RCC, lymph node involvement, and vascular invasion. ${ }^{26}$ Unlike other cancers, the size and number of metastatic lesions, multifocality, and previous disease recurrence, regardless of site, are not significantly correlated with survival. ${ }^{26}$ Therefore, these factors are not contraindications to resection. A few clinical prognostic factors continue to be defined for metastatic RCC. These include the Memorial Sloan-Kettering Cancer Center criteria prognostic score ${ }^{27}$ and the Cleveland Clinic Foundation expanded criteria. The prognostic model developed by Heng et $\mathrm{al}^{28}$ is currently most widely used and has been validated in a large international multicenter dataset. It is similar to the International Database consortium. The International Kidney Cancer working group identified five independent prognostic variables (hemoglobin, white blood cell count, lactate dehydrogenase, alkaline phosphate, and calcium).

The definition of poor prognosis varies between groups. The common features to all variances are shown in Table 2. Interestingly, prognosis was not associated with tumor burden and growth but with tumor biology. As in all cancer therapies, an interdisciplinary approach should be taken, with consideration of tumor biology and response to systemic therapy as a guide for whether surgical resection is appropriate.

\section{Colorectal cancer}

In some cancers, an en-block resection or $\mathrm{R} 0$ resection margin is desired. Patients who undergo R0 resection have the best prognosis following surgery for recurrent rectal cancer and colorectal cancer. With these oncological principals in mind, patients with locally advanced rightside colon cancer that directly invades the duodenum or pancreas can be safely resected with curative potential with minimum morbidity and mortality, and long-term disease-free survival. ${ }^{29}$

Colorectal cancer metastasis usually occurs in the liver and lungs, and rarely to the pancreas. Reddy et $\mathrm{al}^{30}$ reviewed 19 cases of pancreatic resection for colorectal metastasis, and their pooled analysis found that surgery resulted in a

Table 2 Common features of poor prognosis in metastatic renal cell carcinoma

\footnotetext{
I. An interval of less than I year from original to development of metastatic disease

2. Metastases in multiple organs

3. A low Karnofsky performance status

4. Anemia and elevated serum levels of lactate dehydrogenase and calcium

Note: Data from Motzer et $\mathrm{a}^{27}$ and Heng et al. ${ }^{28}$
} 
5 -year survival rate of $27 \%$. Another analysis showed median survival of 1.2 years. ${ }^{31}$ Despite the paucity of cases, there is an evidence that pancreatic resection for colorectal metastasis to the pancreas in conjunction with chemotherapy and biological therapy can improve long-term survival, if a tumorfree resection may be achieved. Thus, if the primary colon cancer is directly invading into the head of the pancreas and duodenum, the recommendation would be to perform a PD with the colon resection. The best outcome would be cases in which there is a response to chemotherapy, a tumor-free resection (R0) is achieved, and the patient is a good candidate for surgery. In addition, a review of 24 studies by Sperti et al demonstrate that pancreatic resection of colorectal metastases provided relief of jaundice and abdominal pain until there is a recurrence. ${ }^{32}$

\section{Melanoma}

Stage IV, metastatic melanoma to gastrointestinal viscera has poor prognosis (AJCC). ${ }^{33}$ There is a case report of six patients with isolated pancreatic metastasis from malignant melanoma who were successfully treated by PD with a prolonged survival of 6 years. ${ }^{34}$

In contrast, in 2011 , Sosman et $\mathrm{al}^{35}$ presented a prospective, multicenter Phase II trial in which they compared 77 patients, 20 of whom had visceral disease. Although the median relapse-free survival was 5 months, they showed that patients with stage IV melanoma achieved prolonged overall survival after complete surgical resection. They could undergo subsequent surgery for isolated recurrence.

Overall, palliative surgery should be offered for symptomatic patients, although this carries the risks of pancreatic surgery. These patients should be carefully selected, and the goals of care and risks of surgery should be compared to their tumor biology and response to other therapies.

\section{Sarcoma}

The most common type of sarcoma is liposarcoma followed by leiomyosarcoma and gastrointestinal tumor (GIST). There are case reports of GIST in pancreas. ${ }^{5}$ GISTs of the pancreas can be managed with imatinib only, or surgery only, or enucleation or neoadjuvant therapy followed by surgery, or adjuvant therapy after surgery or debulking surgery in metastatic or advanced disease. For low-risk GISTs, and after an $\mathrm{R} 0$ resection has been completed, patients could be managed with surveillance. In the case of an R1 or R2 resection or an incomplete resection with positive margins, the patient can be given medical therapy to control the unclear margins. These have all been shown to decrease recurrence and improve prognosis..$^{36,37}$

\section{Gynecologic malignancies}

In surgical management of epithelial ovarian cancer, cytoreduction of advanced stage disease has been shown to improve survival outcomes. ${ }^{38,39}$ Beissel et $\mathrm{al}^{40}$ reported a case in which successful management of a malignant bowel obstruction and achievement of cytoreduction in a stage IIIc papillary serous ovarian cancer required PD.

\section{Conclusion}

Pancreatic metastasectomy is indicated in rare cases where the primary malignancy has had a good response to adjuvant therapy, the tumor biology is favorable, and the patient is a suitable candidate for surgery. In general, a tumor-negative or $\mathrm{R} 0$ resection is the goal. To date, $\mathrm{RCC}$ and colorectal adenocarcinoma through multimodal approaches demonstrate the best disease-free survival.

\section{Trauma}

Pancreatic injury is uncommon and occurs in less than $2 \%$ of all trauma patients and $12 \%$ of patients with abdominal injury. In the USA, penetrating trauma is the cause of the majority of pancreatic injuries, and outside the USA, blunt trauma is the leading cause of injury. ${ }^{41}$ Pancreatic injury is commonly associated with injury to multiple abdominal structures due to its close proximity to solid organs, hollow viscus, and vessels. ${ }^{41}$ In blunt abdominal surgery, $22 \%$ of patients have isolated pancreatic injuries.

The management of blunt pancreatic trauma was reviewed by Potoka et al. ${ }^{42}$ The utility of the Whipple is limited in the setting of blunt and penetrating trauma. Early diagnosis of the presence or absence of main pancreatic duct injury, and the location of the injury is critical, as missed injury after 24 and 48 hours is associated with increasing mortality and morbidity. ${ }^{41}$ In addition, the stability of the patient determines the management of the pancreatic injury. Reports demonstrate that external drainage and distal pancreatectomy result in lower mortality and morbidity rates when compared to pancreaticoenteroanastomoses. ${ }^{42}$

Grade 1-4 pancreatic injuries are not managed with the Whipple procedure or PD. In grade 5 pancreatic injuries, involving massive disruption of the pancreatic head, if the patient is unstable, damage control with wide drainage should be the first step. Their results demonstrated that mortality rates for simpler repair or resection with or without pyloric exclusion were $22.8 \%-25.8 \%$ compared with $46.2 \%$ 
mortality for patients undergoing PD. ${ }^{42} \mathrm{PD}$ is indicated in cases with massive disruption of the pancreatic head with, uncontrollable hemorrhage, massive hemorrhage from adjacent vascular structures, and severe combined duodenal, pancreatic, and biliary injuries. The first operation is to complete the resection, for damage control, and then complete the reconstruction at a second operation. ${ }^{42}$

\section{Pancreatitis}

Acute pancreatitis is a common cause for hospital admission in the USA. Of the 15.9 million clinic visits, from 2009 to 2012 , 274,119 patients were admitted for pancreatitis. ${ }^{43,44}$ The most common risk factors for acute pancreatitis are gallstones, alcohol consumption, and lipid disorders, but the etiology of acute pancreatitis is complex. ${ }^{45}$ Only $3 \%$ of adults who consume large quantities of alcohol will develop alcohol pancreatitis. ${ }^{45}$ Smokers are at risk of chronic pancreatitis. Cigarette smoking is independently associated with chronic pancreatitis. ${ }^{46}$ It is associated with risk of recurrent acute and chronic pancreatitis, because it impairs pancreatic duct cell bicarbonate secretion. ${ }^{45}$ In the first 4 weeks, nonoperative supportive care is indicated. There is no role for PD in acute pancreatitis at this time.

\section{Chronic pancreatitis}

Chronic pancreatitis may lead to diabetes mellitus, malabsorption of food, and chronic pain. Malka et $\mathrm{al}^{47}$ found that the risk of diabetes mellitus does not change whether the patient undergoes surgical or endoscopic decompression. They identified that distal pancreatectomy and pancreatic calcifications were independent risk factors for diabetes mellitus. They found that there were more patients who developed diabetes mellitus after 5 years in the group that underwent distal pancreatectomy, compared to the group that received PD or biliary, cyst, or enteric drainage. Because these procedures did not prevent the onset of diabetes mellitus, and because the onset of calcifications correlated with a 3.2-fold increase of diabetes mellitus, it seems that the risk of diabetes mellitus is due to the progression of chronic pancreatitis and this cannot be prevented with decompression of the pancreatic duct. According to the literature, the incidences of diabetes is $0 \%-22 \%$ at onset, and this increases to more than $80 \%$ after 25 years. ${ }^{47}$

Abdominal pain in chronic pancreatitis may be short episodes or relapsing, or constant and prolonged episodes. Although the studies were not well powered, it was found that patients who were decompressed with surgery had a better pain control compared to decompression using endoscopic techniques $^{48}$ or placebo. In addition, patients with dilated ducts, pain less than 26.5 months, ${ }^{49}$ and have received few drainage procedures, have gotten more complete or partial pain relief ( 2 and 5 years [80\% vs 38\%]) after surgery. Endoscopy can be used as a bridge to definitive surgery. The patients who most benefit from PD have low preoperative pain levels, suggested by the lack of daily opioid use, few endoscopic procedures and have dilated ducts $(8 \mathrm{~mm}$ or greater). ${ }^{50}$ If pain persists, celiac plexus block and pregabalin, or a complete pancreatectomy and islet cell autotransplantation should be considered. ${ }^{51}$

Although low, patients with pancreatitis have an increased risk of developing pancreatic cancer. ${ }^{24}$ Although studies demonstrated that $5 \%$ of patients with chronic pancreatitis may develop pancreatic cancer over a 20 -year period, when following these patents, the possibility of malignancy should not be ruled out. The signs of possible malignancy persistent jaundice, worsening abdominal pain, gastric outlet obstruction and weight loss, and an elevated Ca 19-9 level greater than $300 \mathrm{U} / \mathrm{mL}$ in usually older age adults. In light of this, pancreas resection should be offered to selected and informed patients. In addition, a PD or pancreas resection may simultaneously decompress the pancreatic duct and relieve pain. ${ }^{52}$

\section{Pediatric pancreatic surgery}

Pediatric pancreatic tumors are rare. Independent factors of improved survival are female sex and surgical treatment. ${ }^{53}$ The most common primary pancreatic neoplasms are pancreatoblastoma, solid pseudopapillary neoplasm of the pancreas, and pancreatic endocrine neoplasms/islet cell tumors. Complete surgical resection is the best treatment for primary pancreatic neoplasms. ${ }^{54}$

Pancreatoblastoma occurs in the first decade of life and presents as an incidental abdominal mass. It occurs in the head and tail of the pancreas with equal frequency. Because these tumors secrete alpha-fetoproein, like hepatoblasoma, the treatment is with the same chemotherapeutic agents, and the response to therapy is similar. ${ }^{55}$ This is administered as neoadjuvant therapy to high-stage pancreatoblastoma to optimize a complete resection. ${ }^{55}$ The initial complete resection - primary and metastatic - correlates with long-term survival. . $^{54,55}$

Solitary pseudopapillary neoplasms occur mostly in young female patients. Children present with symptoms, and the lesion is found in the head of the pancreas. ${ }^{54}$ Adults are asymptomatic and the lesions are incidentally found usually in the pancreatic body or tail. Complete surgical resection of any tumor size with negative margins is the treatment of choice and most likely curative. ${ }^{54}$ 
Pancreatic endocrine neoplasms can be sporadic or associated with genetic syndromes such as multiple endocrine neoplasia type-1 (MEN1), Von Hippel-Lindau, neurofibromatosis, and tuberous sclerosis. The prognosis of pancreatic endocrine neoplasms depends on the size, localized or metastatic disease, and histologic criteria. ${ }^{56}$ The most common functioning endocrine tumor is the insulinoma, followed by gastrinoma. ${ }^{56}$ The management of these is described in more detail later in this paper.

Trauma is the most common cause of acute pancreatitis and pancreatic pseudocyst in the pediatric population. ${ }^{42}$ Blunt trauma occurring with motor vehicle crash, pedestrian is struck by a vehicle, handlebar bicycle collisions, child abuse, and falls account for more than $75 \%$ of pancreatic injuries. Unlike in adults, pediatric pancreatic injuries rarely require operative management.

\section{Cancer}

Primary cancers of the head of the pancreas and duodenum are rare and have poor outcomes. Only $20 \%$ of these patients have resectable disease on presentation. Unfortunately, the only chance for cure to date is a complete surgical resection appropriate for the extent and location of the tumor - a PD or Whipple procedure, distal, subtotal, or total pancreatectomy.

\section{Pancreatic cancer}

Candidates who would benefit from surgical resection are patients with no metastatic disease, do not have or only have local (peripancreatic) lymph nodes, no evidence of locally advanced tumor (inseparable from vessel for equal or less than 180 degrees), and no distortion, nor thrombus, nor encasement of the superior mesenteric vein and portal vein. There should be visible tissue planes around the common hepatic artery, gastroduodenal artery, superior mesenteric artery, and celiac axis. If the lesion appears to be resectable and the patient presents with a classical clinical presentation (elevated Ca19-9, dilated pancreatic, and/or biliary duct dilatation due to a stricture), there is no need for the lesion to be biopsied to confirm the diagnosis, and surgical resection can be completed first. If the patient is not a good surgical candidate or if the surgeon believes this is necessary, it is acceptable to perform a percutaneous biopsy. ${ }^{57}$

Borderline resectable patients are those who have no metastatic disease, but due to its location and its association with surrounding vascular structures, the resection is challenging and may require vascular resection and reconstruction. The article written by Lopez et al compares the definition of borderline resectable pancreatic cancer with respect to the relationship of the cancer to the superior mesenteric vein, portal vein, superior mesenteric artery, hepatic artery, and celiac artery as described by MD Anderson, the Americas HepatoPancreato-Biliary Association, the Society for Surgery of the Alimentary Tract, the Society of Surgical Oncology, the National Comprehensive Cancer Network, and Alliance. ${ }^{58}$ In summary, pancreatic cancer is considered a borderline resectable pancreatic cancer if the following radiological findings are present (Table 3). The superior mesenteric vein/portal vein interface to the the tumor is no more than 180 degrees of the vessel wall circumference, and/or there is a short-segment venous occlusion that can be reconstructed. The tumor abuts the superior mesenteric artery of 180 degree or less of vessel wall circumference. The tumor abuts the hepatic artery or there is a short-segment encasement of the tumor to the hepatic artery which is reconstructable. According to the Americas Hepato-Pancreato-Biliary Association, the Society for Surgery of the Alimentary Tract, the Society of Surgical Oncology, the National Comprehensive Cancer Network guidelines, the celiac artery must not be involved, however MD Anderson and Alliance guidelines would consider abutment of the tumor to the celiac artery with a degree of 180 degrees or less of the celiac artery wall circumference as a tumor that is borderline resectable. ${ }^{58}$ These patients might be candidates for neoadjuvant chemotherapy prior to resection, although no formal randomized trials have confirmed its benefit to date. In summary, resectable pancreatic cancer will have a decreased likelihood for the need to reconstruct the vessel, and, preoperative treatments are optional. There is no consensus on definitions of borderline resectable pancreas cancer. Table 3 describes unresectable pancreatic cancer. There is no consensus on definitions of borderline resectable pancreatic cancer.

Staging laparoscopy may be beneficial as a guide for patients with tumor greater than $3 \mathrm{~cm}$, tumors in the neck, body, or tail, or with equivocal computed tomography finding for metastatic disease. ${ }^{59}$

\section{Gallbladder cancer}

Gallbladder cancer invading into the duodenum or pancreas is a stage IIIA to IVA cancer. Unfortunately, less than $25 \%$ of patients present with resectable disease ${ }^{60}$ According to the 2015 International HPB guidelines, these patients should be considered for neoadjuvant chemotherapy for possible reevaluation and resection. ${ }^{60}$

\section{Cholangiocarcinoma}

The annual incidence of cholangiocarcinoma in Western countries is $1-2$ cases per 100,000 . Unfortunately, although the incidence is small, cholangiocarcinoma can 
Table 3 Borderline resectable pancreatic cancer

\begin{tabular}{|c|c|c|c|}
\hline Affected vessel & MD Anderson & AHPBA/SSAT/SSO/NCCN & Alliance \\
\hline $\begin{array}{l}\text { Superior mesenteric vein/ } \\
\text { portal vein }\end{array}$ & Occlusion & $\begin{array}{l}\text { Abutment, impingement, encasement } \\
\text { of the SMV, or short-segment venous } \\
\text { occlusion }\end{array}$ & $\begin{array}{l}\text { Tumor-vessel interface } 180^{\circ} \text { of vessel wall } \\
\text { circumference, and/or reconstructable } \\
\text { occlusion }\end{array}$ \\
\hline Superior mesenteric artery & Abutment & Abutment & $\begin{array}{l}\text { Tumor-vessel interface } 180^{\circ} \text { of vessel wall } \\
\text { circumference }\end{array}$ \\
\hline Hepatic artery & $\begin{array}{l}\text { Abutment or } \\
\text { short-segment } \\
\text { encasement }\end{array}$ & $\begin{array}{l}\text { Abutment or short-segment } \\
\text { encasement }\end{array}$ & $\begin{array}{l}\text { Reconstructable short-segment interface of } \\
\text { any degree between tumor and vessel wall }\end{array}$ \\
\hline Celiac artery & Abutment & Uninvolved & $\begin{array}{l}\text { Tumor-vessel interface } 180^{\circ} \text { of vessel wall } \\
\text { circumference }\end{array}$ \\
\hline
\end{tabular}

Notes: Reproduced from Lopez NE, Prendergast C, Lowy AM. Borderline resectable pancreatic cancer: definitions and management. World J Gastroenterol. 20I4;20(3I): 10740-1075I. Copyright (C) 2014 Baishideng Publishing Group Inc. All rights reserved. ${ }^{58}$

Abbreviations: SMV, superior mesenteric vein; AHPBA, Americas Hepato-Pancreato-Biliary Association; SSAT, Society for Surgery of the Alimentary Tract; SSO, Society of Surgical Oncology; NCCN, National Comprehensive Cancer Network.

infiltrate adjacent vessels, becoming unresectable. Tumor differentiation and nodal status are important prognostic factors. ${ }^{61} \mathrm{PD}$ with lymphadenectomy remains the procedure of choice to obtain cancer-free surgical margins and to harvest enough lymph nodes for staging. ${ }^{62}$ Without resection, the 5 -year survival is less than $10 \%$. If resected, 5 -year survival is $23 \%-33 \% .{ }^{61}$ When intrahepatic, perihilar, and distal cholangiocarcinoma were compared, the presence of microvascular invasion had the strongest correlation with decreased survival in all three types.

\section{Duodenal cancer}

The 5-year survival rate for resectable cases is $63 \% .^{63}$ The procedure of choice for the treatment of duodenal carcinoma is a Whipple with lymphadenectomy. Because the relationship between the extent of lymph node metastasis and tumor size and the impact this may have on prognosis is unclear, a disease with lymphadenopathy is not a contraindication to surgery, and these patients should be offered resection with lymphadenectomy. ${ }^{63}$ These patients should then be offered chemotherapy postresection. ${ }^{63}$ Superficial nonampullary duodenal epithelial tumors, including high-grade adenoma, should be treated endoscopically or surgically. For low-risk lesions, endoscopic mucosal resection may be reasonable; however, surgery remains the standard method for lesions that are technically impossible to remove by endoscopy. ${ }^{63}$ The Spigelman classification is usually followed to determine which patients with hereditary disease and with duodenal polyps become candidates for PD (Table 4) or continued endoscopic management.

An estimated $65 \%-90 \%$ of adenocarcinomas arise from adenomas. ${ }^{64}$ The risk of lymph node disease correlates with size of the primary tumor: $>1 \mathrm{~cm}=9 \%, 1-1.5 \mathrm{~cm}=25 \%$, $>1.5 \mathrm{~cm}=40 \%-50 \% .{ }^{64}$ Approximately $30 \%-70 \%$ of patients diagnosed with familial adenomatous polyposis (FAP) have duodenal adenomas. ${ }^{64}$ By age 57 , the cumulative incidence
Table 4 Spigelman classification and risk of duodenal adenocarcinoma

\begin{tabular}{llll}
\hline Variables & I point & 2 points & 3 points \\
\hline Number of polyps & I-4 & $5-20$ & $>20$ \\
Polyp size $(\mathrm{mm})$ & I-4 & $5-10$ & $>10$ \\
Histology & Tubulous & Tubulovillous & Villous \\
Dysplasia & Mild & Moderate & Severe \\
Spigelman score & Stage & Surveillance & Risk of adenocarcinoma \\
0 & 0 & Every 4 years & - \\
I-4 & I & Every 4 years & - \\
$5-6$ & II & $2-3$ years & $2.3 \%$ \\
$7-8$ & III & I-4 years & $2.4 \%$ \\
$9-12$ & IV & Surgery & $36 \%$ \\
\hline
\end{tabular}

Note: Data from Spigelman et al. ${ }^{74}$

rate of duodenal cancer is $4.5 \%$, and the median age of the development of duodenal carcinoma is 52 years. ${ }^{64}$ On average, there is a 22-year interval between colectomy for FAP and upper gastrointestinal cancer development. ${ }^{64} \mathrm{FAP}$ patients begin scoping surveillance at 25-30 years of age and staging is done using Spiegelman classification (Table 5).

\section{Ampullary cancer}

Ampullary carcinoma arises from the ampulla or papilla of Vater. There are two histological types: intestinal and pancreatobiliary. ${ }^{65}$ The intestinal type has a better prognosis. A few studies demonstrate that administration of packed red blood cells, increased tumor stage, substantial invasion depth of the tumor, histological grading, positive resection border, vascular and lymphatic vessel invasion, and Ca19-9 levels higher than $37 \mathrm{U} / \mathrm{L}$ were each associated with a significantly reduced long-term survival $1{ }^{65}$ There is currently no significant difference in overall survival between patients who undergo a pylorus-preserving PD or a classic Whipple with a standard lymphadenectomy. Owing to the location of these lesions, the patients present with symptoms earlier at the time of diagnosis, and these lesions have a high rate of being suc- 
Table 5 Unresectable Pancreatic Cancer

I) Metastatic disease

a. Distant metastases

b. Metastatic lymphadenopathy beyond limits of resection

2) Locally unresectable tumor

a. Invasion to inferior vena cava (IVC)

b. Occlusion of SMV and PV, and not reconstructable

c. Encasement of HA and gastroduodenal artery and not recontructable

d. Encasement of the SMA and CA

3) Unfit patient for abdominal surgery

Note: Data from Clancy. ${ }^{13}$

Abbreviations: SMV, superior mesenteric vein; PV, portal vein; SMA, superior mesenteric artery; CA, celiac artery; HA, hepatic artery.

cessfully resected. Because there is a lower risk of invasion, these patients should be offered a PD and lymphadenectomy even with positive lymph nodes.

In high-risk operative patients, it is possible to perform an endoscopic resection, but in resection without cleared margins, the survival time is severely reduced. If the resection margin contains microscopic evidence of cancer of lymphatic invasion, the patient should be offered chemo and radiation therapies. Unfortunately, the biopsy-negative rate is $40 \%-85 \%,{ }^{65}$ as the lesion may appear benign.${ }^{65}$ If this is the case, endoscopic resection may be offered first, followed by evaluation of the larger specimen with the submucosal tissue. If there is a high suspicion of cancer, and the patient is a good operative candidate, an operation should be offered.

\section{Neuroendocrine tumor}

There are nonfunctional and functional pancreatic neuroendocrine tumors (pNETs). Nonfunctional pNETs usually present with abdominal pain (60\%), weight loss (25\%-35\%), jaundice $(17 \%-50 \%)$, and palpable mass $(7 \%-40 \%)$. If the lesion is more than $2 \mathrm{~cm}$, surgical resection may be either local resection, or multivisceral resection of locally advanced tumor. If the lesion is smaller than $2 \mathrm{~cm}$, one may observe, or resect by enucleation or parenchymal sparing procedures. There is no difference in survival benefit with resection with a Whipple or enucleation. ${ }^{66}$

Functional pNETs are insulinoma, gastrinoma, glucagonoma, somatostatinoma, and VIPoma. Unlike the other neuroendocrine tumors, insulinomas are not easily detectable on somatostatin (octreotide) scan. Patients with glucagonoma, somatostatinoma, and VIPoma should be offered surgical resection with lymphadenectomy. Insulinomas and gastrinomas, if smaller than $2 \mathrm{~cm}$ or located at the head of the pancreas, should be considered for enucleation and localized resections. Surgical resection of gastrinoma has demonstrated a decrease in the rate of metastatic development and extended survival. ${ }^{66}$

\section{MEN I}

MEN1 (inactivation mutation of tumor suppressor) is an autosomal dominant condition in which patients frequently present with hyperparathyroidism, pancreatic endocrine tumors (PETs), and pituitary tumors. ${ }^{67}$ PETs in MEN1 patients have an earlier disease onset, a higher risk of malignancy, and present with multiple lesions distributed through the pancreas and duodenum. Unlike sporadic PETs, these PETs vary in hormone production, and they also have an inactivated germ line mutation in the MEN1 tumor suppressor gene.

The goal of surgical treatment of these PETs is to prevent malignant tumor progression and to treat the clinical symptoms. MEN1 patients usually present with primary hyperparathyroidism first, and a total parathyroidectomy followed by parathyroid autoimplantation in patients with MEN1 reduces the calcium and PTH, as well as decreases the secretion of other hormones such as gastrin. This should be completed before the PETs are addressed, except if the PET is an insulinoma.

MEN1 patients can present with nonfunctioning and functioning PETs. Nonfunctioning PETs and thymic carcinomas are frequent causes of death, because when they are diagnosed, they are usually malignant and larger. ${ }^{67}$ Tumor size correlates with malignancy, and lesions $>2.0 \mathrm{~cm}$ have a risk of malignancy. ${ }^{68}$ Tumors $>1.0 \mathrm{~cm}$ have a risk of metastasizing to the liver. ${ }^{68}$ Surgical resection is recommended with extended distal pancreatectomy associated with enucleation of PETs $>1 \mathrm{~cm}$ located at the pancreatic head to prevent liver metastasis, or total pancreatectomy with or without duodenectomy. However, the total pancreatectomy decreases the patient's quality of life as it leaves them with brittle diabetes mellitus. ${ }^{68}$

In MEN1 patients who develop gastrinomas, usually multiple, asynchronous tumors are found in the duodenum more frequently and less often in the pancreas. Approximately $60 \%$ are malignant and associated with multiple gastric carcinoids. ${ }^{67}$ These lesions have an unpredictable course, are difficult to locate preoperatively, and are multiple in the duodenum. Gastrinomas present indolently with or without metastasis or rapid tumor progression. The relative aggressiveness of these tumors can be determined with gastrin levels, histological differentiation, Ki-67 positivity, mitotic number, and the presence of progesterone receptors. ${ }^{68}$ There is controversy as to whether surgical treatment should be reserved for gastrinomas $>3 \mathrm{~cm}$ only, or whether aggressive and early surgical intervention is indicated as soon as diagnosis is made. ${ }^{68}$ This would include limited surgical resection with excision of duodenal tumors, excision of pancreatic cephalic lesion, and distal pancreatectomy with 
lymphadenectomy, or alternatively, PD with regional lymphadenectomy. ${ }^{68}$ Although there are functional advantages to pylorus-preserving techniques, duodenal tumors are found in the first 1-2 cm of duodenum, and it is safer to perform a classic Whipple in these patients with gastrinomas.

Due to difficulties in detecting the lesion, a total pancreatectomy may be the preferred treatment. On the other hand, insulinomas in sporadic cases can be managed with enucleation if it is not associated with the main duct and if it is $<2 \mathrm{~cm}$. However, in MEN1 patients, the first treatment is distal pancreatectomy to the mesenteric vein and enucleation of lesions in the head of the pancreas. If it involves the pancreatic head extensively, pylorus-preserving PD is the treatment of choice. There is a higher recurrence rate in MEN1 versus sporadic cases. Glucagonomas, somatostatinomas, and VIPomas are usually malignant and often are larger than $3 \mathrm{~cm}$; so radical surgical resection is the proposed treatment.

Surgical strategies ${ }^{67}$ to manage PETs in patients with MEN1 are different from those applied to patients with sporadic PETs. Because of these reasons, clinical and genetic diagnosis of MEN1 mutation should be determined before surgery in all patients presenting with apparently sporadic PETs.

\section{Cystic lesions of the pancreas} Intraductal papillary mucinous neoplasms Intraductal papillary mucinous neoplasms (IPMNs) are cystic, intraductal, and mucin-producing tumors with dysplastic cells, which range from benign adenoma to invasive carcinoma. The majority of patients present with abdominal pain, obstructive jaundice, or pancreatitis, or weight loss. These IPMNs may be main duct, branch duct, and mixed or combined. Main duct IPMNs have a higher risk of progression to malignancy at presentation and these should be resected. Branch IPMNs are benign and have a lower risk of malignancy. These can be observed. All main duct IPMNs should be resected. The high risk stigmata of malignancy includes: 1) obstructive jaundice with cystic lesion of the pancreatic head; 2) cyst with enhancing solid component; and 3) main pancreatic duct size is equal or greater than $10 \mathrm{~mm}$, and an elevated Ca 19-9. Surgery should be considered in patients with these findings. Further investigations such as the use of endoscopic ultrasound (EUS) to further evaluate branch duct IPMN in patients with clinical pancreatitis, and on imaging, have: 1) cysts $\geq 3 \mathrm{~cm}$; 2) thickened or enhancing cystic wall; 3) main duct size of 5 to $9 \mathrm{~mm}$; 4) nonenhancing mural modules; and/or 5) abrupt changes in the caliber of pancreatic duct with distal pancreatic atrophy, and elevated $\mathrm{Ca}$ 19-9 levels. On EUS and fine needle aspiration of the cyst, if there are mural nodules, main duct involvement with thickened walls, intraductal mucin or mural nodules, or cytology suspicious or positive for malignancy and elevated Ca 19-9 levels, these patients should be considered for surgery. If the main duct is not seen, this is inconclusive. ${ }^{69-71}$ If there are no suspicious features, according to the International Consensus Guidelines 2012, it is possible to follow the management of branch duct IPMN according to the size of the largest cyst. ${ }^{69}$ If the cyst is smaller than $1 \mathrm{~cm}$, the patient should be followed with a repeat CT/MRI in 2 to 3 years. If the cyst is between 1 and $2 \mathrm{~cm}$, the patient should have an annual CT/MRI for 2 years, then the interval can be lengthened if there are no changes. If the cyst is between 2 and $3 \mathrm{~cm}$ in size, the patient should have an EUS in 3 to 6 months, then lengthen interval alternating MRI with EUS as appropriate. Fit patients who will need prolonged surveillance may be considered for surgery. If the cyst is larger than $3 \mathrm{~cm}$, the patient should be surveillance alternating MRI and EUS every 3 to 6 months. Fit patients should be considered for surgery.

\section{Mucinous cystic neoplasms}

Mucinous cystic neoplasms usually present as solitary cystic lesion of the body or tail of the pancreas in middle-aged women, without ductal communication. They have an 18\%-48\% potential for malignant transformation. Because of the risk of malignancy and progression to invasive carcinoma, young and healthy patients should be offered a resection. If they have abdominal pain, this could indicate resection. Mural nodules and arterial enhancement are suggestive of malignancy, and these should be removed. Resection may be curative for invasive cystadenocarinoma. ${ }^{72}$ Five-year survival for patients undergoing $\mathrm{R} 0$ resection for cystoadenocarcinoma is $60 \%{ }^{72}$

\section{Serous cystadenoma}

Serous cystadenomas are serous cystic neoplasms usually found in older women. They have a less than $1 \%$ chance of malignant transformation. They present with abdominal pain, palpable mass, or jaundice. They occur sporadically and in the setting of people with Von Hippel-Lindau disease. Resection is recommended if the lesions cause pain or jaundice and are growing, or if the nodule is larger than $4 \mathrm{~cm} .{ }^{70}$ Serial surveillance is indicated for asymptomatic lesions. Considerations for resection include age, number of years after diagnosis, oligocystic morphology, and history of other malignancy. Lesions $>4 \mathrm{~cm}$ grow faster than those $<4 \mathrm{~cm}$.

Cystic pancreatic endocrine neoplasias are rare and potentially malignant tumors. They have enhancing walls on computed tomography scan. Because these have a low risk of 
invasive disease, discussion evolves around nonanatomical versus anatomical resection with lymphadenectomy to aid in detecting nodal disease and establish prognosis. ${ }^{71}$

\section{Solid pseudopapillary neoplasm}

This occurs almost only in young women. Because $10 \%-20 \%$ of cases demonstrate malignant behavior, management with surgical resection is recommended. More than $80 \%$ of solid pseudopapillary neoplasm patients experience long-term survival after surgery. ${ }^{73}$

\section{Conclusion}

As new treatments arise and chronic diseases are better managed, age is no longer a contraindication to being a candidate for a Whipple. To date, the best treatment and chance for cure of malignant diseases of the pancreatic head, duodenum, and distal common bile duct is early detection and a PD. All patients should be evaluated by a hepatopancreatobiliary surgeon, and the goals of the treatment should be discussed and agreed upon to achieve selection of the most appropriate patients and to obtain the best outcomes.

\section{Acknowledgment}

The authors would like to thank Dr John May and Jennifer Victory with their assistances in preparing this manuscript.

\section{Disclosure}

The authors report no conflicts of interest in this work.

\section{References}

1. Whipple AO. Pancreaticoduodenectomy for islet carcinoma: a five-year follow-up. Ann Surg. 1945;121(6):847-852.

2. Hsu CC, Wolfgang CL, Laheru DA, et al. Early mortality risk score: identification of poor outcomes following upfront surgery for resectable pancreatic cancer. J Gastrointest Surg. 2012;16(4):753-761.

3. Kimura W, Miyata H, Gotoh M, et al. A pancreaticoduodenectomy risk model derived from 8,575 cases from a national single-race population (Japanese) using a web-based data entry system: the 30-day and in-hospital mortality rates for pancreaticoduodenectomy. Ann Surg. 2014;259(4):773-780.

4. Greenblatt DY, Kelly KJ, Rajamanickam V, et al. Preoperative factors predict perioperative morbidity and mortality after pancreaticoduodenectomy. Ann Surg Oncol. 2011;18(8):2126-2135.

5. Beltrame V, Gruppo M, Pastorelli D, Pedrazzoli S, Merigliano S, Sperti C. Outcome of pancreaticoduodenectomy in octogenarians: single institution's experience and review of the literature. J Visc Surg. 2015;152(5):279-284.

6. Langan RC, Huang CC, Colton S, et al. Readmissions after major cancer surgery among older adults. Surgery. 2015;158(2):428-437.

7. Langan RC, Huang CC, Mao WR, et al. Pancreaticoduodenectomy hospital resource utilization in octogenarians. Am J Surg. 2016;211(1): 70-75.

8. Zogg CK, Mungo B, Lidor AO, Stern M, Rios Diaz AJ, Halder AH, Molena D. Influence of body mass index on outcomes after major resection for cancer. Surgery. 2015;158(2):472-485.
9. El Nakeeb A, Hamed H, Shehta A, et al. Impact of obesity on surgical outcomes post-pancreaticoduodenectomy: a case-control study. Int $J$ Surg. 2014;12(5):488-493.

10. Mathur A, Luberice K, Paul H, Franka C, Rosemurgy A. Increasing body mass index portends abbreviated survival following pancreatoduodenectomy for pancreatic adenocarcinoma. Am J Surg. 2015;209(6):969-973.

11. House MG, Fong Y, Arnaoutakis DJ, et al. Preoperative predictors for complications after pancreaticoduodenectomy: impact of BMI and body fat distribution. J Gastrointest Surg. 2008;12(2):270-278.

12. Pausch T, Hartwig W, Hinz U, et al. Cachexia but not obesity worsens the postoperative outcome after pancreatoduodenectomy in pancreatic cancer. Surgery. 2012;152(3 Suppl 1):S81-S88.

13. Clancy TE. Surgery for pancreatic cancer. Hematol Oncol Clin North Am. 2015;29(4):701-716.

14. Colquitt JL, Pickett K, Loveman E, Frampton GK. Surgery for weight loss in adults. Cochrane Database Syst Rev. 2014;8:CD003641.

15. Hatzaras I, Sachs TE, Weiss M, Wolfgang CL, Pawlik TM. Pancreaticoduodenectomy after bariatric surgery: challenges and available techniques for reconstruction. J Gastrointest Surg. 2014;18(4):869-877.

16. Jimenez RE, Warshaw AL, Rattner DW, Willett CG, McGrath D, Fernandez-del Castillo C. Impact of laparoscopic staging in the treatment of pancreatic cancer. Arch Surg. 2000;135(4):409-414; discussion 414-415.

17. Hennig R, Tempia-Caliera AA, Hartel M, Buchler MW, Friess H. Staging laparoscopy and its indications in pancreatic cancer patients. Dig Surg. 2002;19(6):484-488.

18. Vollmer CM, Drebin JA, Middleton WD, et al. Utility of staging laparoscopy in subsets of peripancreatic and biliary malignancies. Ann Surg. 2002;235(1):1-7.

19. Karachristos A, Scarmeas N, Hoffman JP. CA 19-9 levels predict results of staging laparoscopy in pancreatic cancer. J Gastrointest Surg. 2005;9(9):1286-1292.

20. Liang S, Hameed U, Jayaraman S. Laparoscopic pancreatectomy: indications and outcomes. World J Gastroenterol. 2014;20(39): 14246-14254.

21. Campa D, Rizzato C, Capurso G, et al. Genetic susceptibility to pancreatic cancer and its functional characterisation: the PANcreatic Disease ReseArch (PANDoRA) consortium. Dig Liver Dis. 2013;45(2):95-99.

22. Canto MI, Harinck F, Hruban RH, et al. International cancer of the pancreas screening (CAPS) consortium summit on the management of patients with increased risk for familial pancreatic cancer. Gut. 2013;62(3):339-347.

23. McBride KA, Ballinger ML. Li-Fraumeni syndrome: cancer risk assessment and clinical management. Nat Rev Clin Oncol. 2014; 11(5):260-271.

24. McKay CJ, Glen P, McMillan DC. Chronic inflammation and pancreatic cancer. Best Pract Res Clin Gastroenterol. 2008;22(1):65-73.

25. Zerbi A, Pecorelli N. Pancreatic metastases: an increasing clinical entity. World J Gastrointest Surg. 2010;2(8):255-259.

26. Kroeger N, Xie W, Lee JL, et al. Metastatic non-clear cell renal cell carcinoma treated with targeted therapy agents: characterization of survival outcome and application of the International mRCC Database Consortium criteria. Cancer. 2013;119(16):2999-3006.

27. Motzer RJ, Mazumdar M, Bacik J, Berg W, Amsterdam A, Ferrara J. Survival and prognostic stratification of 670 patients with advanced renal cell carcinoma. J Clin Oncol. 1999;17(8):2530-2540.

28. Heng DY, Xie W, Regan MM, et al. External validation and comparison with other models of the International Metastatic Renal-Cell Carcinoma Database Consortium prognostic model: a population-based study. Lancet Oncol. 2013;14(2):141-148.

29. Lee WS, Lee WY, Chun HK, Choi SH. En bloc resection for right colon cancer directly invading duodenum or pancreatic head. Yonsei Med J. 2009;50(6):803-806.

30. Reddy S, Wolfgang CL. The role of surgery in the management of isolated metastases to the pancreas. Lancet. 2009;10:287-293.

31. Konstantinidis IT, Dursun A, Zheng H, et al. Metastatic tumors in the pancreas in the modern era. J Am Coll Surg. 2010;211(6):749-753. 
32. Sperti C, Moletta L, Patane G. Metastatic tumors to the pancreas: The role of surgery. World J Gastrointest Oncol. 2014;6(10):381-392.

33. Tas F. Metastatic behavior in melanoma: timing, pattern, survival, and influencing factors. Journal of Oncology. 2012.

34. Birnbaum DJ, Moutardier V, Turrini O, Goncalves A, Delpero JR. Isolated pancreatic metastasis from malignant melanoma: is pancreatectomy worthwile? J Surg Tech Case Rep. 2013;5(2):82-84.

35. Sosman JA, Moon J, Tuthill RJ, et al. A Phase 2 trial of complete resection for stage IV melanoma: results of Southwest Oncology Group Clinical Trial S9430. Cancer. 2011;117(20):4740-4746.

36. Duffaud F, Meeus P, Bachet JB, et al. Conservative surgery vs duodeneopancreatectomy in primary duodenal gastrointestinal stromal tumors (GIST): a retrospective review of 114 patients from the French sarcoma group (FSG). Eur J Surg Oncol. 2014;40(10):1369-1375.

37. Colombo C, Ronellenfitsch U, Yuxin Z, et al. Clinical, pathological and surgical characteristics of duodenal gastrointestinal stromal tumor and their influence on survival: a multi-center study. Ann Surg Oncol. 2012;19(11):3361-3367.

38. Aletti GD, Dowdy SC, Gostout BS, et al. Aggressive surgical effort and improved survival in advanced-stage ovarian cancer. Obstet Gynecol. 2006;107(1):77-85.

39. Eisenhauer EL, Abu-Rustum NR, Sonoda Y, Aghajanian C, Barakat RR, Chi DS. The effect of maximal surgical cytoreduction on sensitivity to platinum-taxane chemotherapy and subsequent survival in patients with advanced ovarian cancer. Gynecol Oncol. 2008;108(2): 276-281.

40. Beissel JM, Kendrick ML, Podratz KC, Bakkum-Gamez JN. Pancreaticoduodenectomy in optimal primary cytoreduction of epithelial ovarian cancer: a case report and review of the literature. Gynecol Oncol Rep. 2014; 10:25-27.

41. Biffl WL, Moore EE, Croce M, et al. Western trauma association critical decisions in trauma: management of pancreatic injuries. J Trauma Acute Care Surg. 2013;75(6):941-946.

42. Potoka DA, Gaines BA, Leppaniemi A, Peitzman AB. Management of blunt pancreatic trauma: what's new? Eur J Trauma Emerg Surg. 2015;41(3):239-250.

43. Peery AF, Crockett SD, Barritt AS, et al. Burden of gastrointestinal, liver, and pancreatic diseases in the United States. Gastroenterology. 2015;149(7):1731-1741.e3.

44. Peery AF, Dellon ES, Lund J, et al. Burden of gastrointestinal disease in the United States: 2012 update. Gastroenterology. 2012;143(5):1179-1187. e1171-e1173.

45. Greer JB, Thrower E, Yadav D. Epidemiologic and mechanistic associations between smoking and pancreatitis. Curr Treat Options Gastroenterol. 2015;13(3):332-346.

46. Law R, Parsi M, Lopez R, Zuccaro G, Stevens T. Cigarette smoking is independently associated with chronic pancreatitis. Pancreatology. 2010;10(1):54-59.

47. Malka D, Hammel P, Sauvanet A, et al. Risk factors for diabetes mellitus in chronic pancreatitis. Gastroenterology. 2000;119(5): 1324-1332.

48. Ahmed Ali U, Pahlplatz JM, Nealon WH, van Goor H, Gooszen HG, Boermeester MA. Endoscopic or surgical intervention for painful obstructive chronic pancreatitis. Cochrane Database Syst Rev. 2015;3:CD007884.

49. Yang CJ, Bliss LA, Freedman SD, et al. Surgery for chronic pancreatitis: the role of early surgery in pain management. Pancreas. 2015;44(5):819-823.

50. van der Gaag NA, van Gulik TM, Busch OR, et al. Functional and medical outcomes after tailored surgery for pain due to chronic pancreatitis. Ann Surg. 2012;255(4):763-770.

51. Wilson GC, Sutton JM, Smith MT, et al. Total pancreatectomy with islet cell autotransplantation as the initial treatment for minimal-change chronic pancreatitis. HPB (Oxford). 2015;17(3):232-238.

52. Raimondi S, Lowenfels AB, Morselli-Labate AM, Maisonneuve P, Pezzilli R. Pancreatic cancer in chronic pancreatitis; aetiology, incidence, and early detection. Best Pract Res Clin Gastroenterol. 2010;24(3):349-358.
53. Perez EA, Gutierrez JC, Koniaris LG, Neville HL, Thompson WR, Sola JE. Malignant pancreatic tumors: incidence and outcome in 58 pediatric patients. J Pediatr Surg. 2009;44(1):197-203.

54. Nasher O, Hall NJ, Sebire NJ, de Coppi P, Pierro A. Pancreatic tumours in children: diagnosis, treatment and outcome. Pediatr Surg Int. 2015;31(9):831-835.

55. Bien E, Godzinski J, Dall'igna P, et al. Pancreatoblastoma: a report from the European cooperative study group for paediatric rare tumours (EXPeRT). Eur J Cancer. 2011;47(15):2347-2352.

56. Park M, Koh KN, Kim BE, Im HJ, Kim DY, Seo JJ. Pancreatic neoplasms in childhood and adolescence. J Pediatr Hematol Oncol. 2011;33(4):295-300.

57. Harewood GC, Wiersema MJ. Endosonography-guided fine needle aspiration biopsy in the evaluation of pancreatic masses. Am J Gastroenterol. 2002;97(6):1386-1391.

58. Lopez NE, Prendergast C, Lowy AM. Borderline resectable pancreatic cancer: definitions and management. World J Gastroenterol. 2014;20(31):10740-10751.

59. Katz MH, Marsh R, Herman JM, et al. Borderline resectable pancreatic cancer: need for standardization and methods for optimal clinical trial design. Ann Surg Oncol. 2013;20(8):2787-2795.

60. Aloia TA, Jarufe N, Javle M, et al. Gallbladder cancer: expert consensus statement. HPB (Oxford). 2015;17(8):681-690.

61. Ercolani G, Dazzi A, Giovinazzo F, et al. Intrahepatic, peri-hilar and distal cholangiocarcinoma: three different locations of the same tumor or three different tumors? Eur J Surg Oncol. 2015;41(9):1162-1169.

62. Andrianello S, Paiella S, Allegrini V, et al. Pancreaticoduodenectomy for distal cholangiocarcinoma: surgical results, prognostic factors, and long-term follow-up. Langenbecks Arch Surg. 2015;400(5):623-628. Dutch.

63. Kato Y, Takahashi S, Kinoshita T, Shibasaki H, Gotohda N, Konishi M. Surgical procedure depending on the depth of tumor invasion in duodenal cancer. Jpn J Clin Oncol. 2014;44(3):224-231.

64. Brosens LA, Keller JJ, Offerhaus GJ, Goggins M, Giardiello FM. Prevention and management of duodenal polyps in familial adenomatous polyposis. Gut. 2005;54(7):1034-1043.

65. Klein F, Jacob D, Bahra M, et al. Prognostic factors for long-term survival in patients with ampullary carcinoma: the results of a 15-year observation period after pancreaticoduodenectomy. HPB Surg. 2014;2014:970234.

66. Kulke MH, Anthony LB, Bushnell DL, et al. NANETS treatment guidelines: well-differentiated neuroendocrine tumors of the stomach and pancreas. Pancreas. 2010;39(6):735-752.

67. Machado MC. Surgical treatment of pancreatic endocrine tumors in multiple endocrine neoplasia type 1. Clinics. 2012;67(Suppl 1):145-148.

68. Imamura M. Recent standardization of treatment strategy for pancreatic neuroendocrine tumors. World J Gastroenterol. 2010;16(36):4519-4525.

69. Tanaka M, Fernandez-del Castillo C, Adsay V, et al. International consensus guidelines 2012 for the management of IPMN and MCN of the pancreas. Pancreatology. 2012;12(3):183-197.

70. Kim TS, Fernandez-del Castillo C. Diagnosis and management of pancreatic cystic neoplasms. Hematol Oncol Clin North Am. 2015;29(4):655-674.

71. Tseng JF, Warshaw AL, Sahani DV, Lauwers GY, Rattner DW, Fernandezdel Castillo C. Serous cystadenoma of the pancreas: tumor growth rates and recommendations for treatment. Ann Surg. 2005;242(3):413-419; discussion 419-421.

72. Kaimakliotis P, Riff B, Pourmand K, et al. Sendai and fukuoka consensus guidelines identify advanced neoplasia in patients with suspected mucinous cystic neoplasms of the pancreas. Clin Gastroenterol Hepatol. 2015;13(10):1808-1815.

73. Butte JM, Brennan MF, Gonen M, et al. Solid pseudopapillary tumors of the pancreas. Clinical features, surgical outcomes, and long-term survival in 45 consecutive patients from a single center. $J$ Gastrointest Surg. 2011;15(2):350-357.

74. Spigelman AD, Williams CB, Talbot IC, Domizio P, Phillips RK. Upper gastrointestinal cancer in patients with familial adenomatous polyposis. Lancet. 1989;2(8666):783-785. 
Open Access Surgery

\section{Publish your work in this journal}

Open Access Surgery is an international, peer-reviewed, open access journal that focuses on all aspects of surgical procedures and interventions. Patient care around the peri-operative period and patient outcomes post surgery are key topics. All grades of surgery from minor cosmetic interventions to major surgical procedures are covered. Novel techniques

and the utilization of new instruments and materials, including implants and prostheses that optimize outcomes constitute major areas of interest. The manuscript management system is completely online and includes a very quick and fair peer-review system. Visit http://www.dovepress.com/ testimonials.php to read real quotes from published authors. 